\title{
RETRACTED ARTICLE: Apoptotic suppression of inflammatory macrophages and foam cells in vascular tissue by miR-23a
}

\section{Magdalena Stoeva ${ }^{1}$}

Published online: 31 January 2019

(C) IUPESM and Springer-Verlag GmbH Germany, part of Springer Nature 2019

This article [1] is retracted at the request of Editor-in-Chief because its content has been duplicated from an unpublished manuscript authored by Yu Qiao, Chuxuan Wang, Ning Huang, Nazhen Dong, Dong Han, Iujing Wang, Dantong Wu, Xuemei Zhang, Qian Sun, Jing Luan, Xinlei Wang, Peihua Cao, Xu Gao, Ning Ma and Shanshun Luo without permission. All authors agree to this retraction.

[1] Roufayel, R. \& Kadry, S. Health Technol. (2019). https:// doi.org/10.1007/s12553-019-00301-y 